\title{
Azilsartan/chlorthalidone combination therapy for blood pressure control
}

\author{
This article was published in the following Dove Press journal: \\ Integrated Blood Pressure Control \\ 24 May 2013 \\ Number of times this article has been viewed
}

\section{Judy WM Cheng}

Massachusetts College of Pharmacy and Health Sciences, Brigham and Women's Hospital, Boston, MA, USA
Background: Edarbyclor ${ }^{\circledR}$ is a combined angiotensin receptor blocker (ARB) and thiazide-like diuretic (azilsartan and chlorthalidone), and was approved on December 20, 2011 by the US Food and Drug Administration (FDA) for hypertension management.

Objective: To review the pharmacology, pharmacokinetics, efficacy, safety, tolerability, and role of azilsartan plus chlorthalidone for hypertension management.

Methods: Peer-reviewed clinical trials, review articles, and relevant treatment guidelines, were identified from the databases MEDLINE and Current Contents (both 1966 to February 15, 2013, inclusive) using search terms "azilsartan", "chlorthalidone", "pharmacology", "pharmacokinetics", "pharmacodynamics", "pharmacoeconomics", and "cost-effectiveness". The FDA website, as well as manufacturer prescribing information, was also reviewed to identify other relevant information.

Results: Azilsartan is a new ARB with high affinity for the angiotensin 1 receptor, approved by the FDA for hypertension management. Unlike other ARBs, azilsartan has no clinical data supporting improvement in cardiovascular outcomes, and is not approved for indications other than hypertension, which a select few other ARBs may be used for (eg, diabetic nephropathy and heart failure). Chlorthalidone is a longer acting thiazide-like diuretic that has been demonstrated to improve cardiovascular outcomes. Combination treatment with azilsartan/chlorthalidone is effective for reducing blood pressure. Compared to olmesartan/hydrochlorothiazide and azilsartan/hydrochlorothiazide combinations, azilsartan/chlorthalidone appears to be more efficacious for reducing blood pressure.

Conclusions: Azilsartan/chlorthalidone can be considered an antihypertensive therapy option in patients for whom combination therapy is required (blood pressure $>20 \mathrm{mmHg}$ systolic or $>10 \mathrm{mmHg}$ diastolic above goal). Cost to patients and insurance coverage will probably determine whether azilsartan/chlorthalidone will be the most appropriate combination therapy for an individual patient.

Keywords: azilsartan, chlorthalidone, hypertension

\section{Introduction}

The Seventh Report of the Joint National Committee on Prevention, Detection, Evaluation, and Treatment of High Blood Pressure (JNC7) defines hypertension as blood pressure (BP) greater than $140 / 90 \mathrm{mmHg}$ in adults 18 years and older. ${ }^{1}$ According to the National Health and Nutrition Examination Survey (NHANES) 2003-2006, 33.6\% of Americans (74.5 million people) $\geq 20$ years of age have hypertension. ${ }^{2}$ Of these patients diagnosed with hypertension, 78\% are aware of their diagnosis, 68\% are taking antihypertensive medication, and despite knowledge gained from large clinical trials regarding hypertension management, only $44 \%$ of patients treated were well controlled. In 2010 , it was estimated that the cost to manage hypertension in the US averaged near 76.6 billion in health care services, medications, and missed days of work. ${ }^{2}$ Hypertension is a major risk factor 
for coronary heart disease, stroke, heart failure, and kidney disease. ${ }^{3}$ Thus, the ultimate goal for hypertension management is to reduce overall cardiovascular and renal morbidity and mortality. ${ }^{1}$ This lack of medical success in optimally controlling $\mathrm{BP}$ is among one of the many reason why new antihypertensive agents continue to be developed.

The current JNC7 guidelines recommend administering more than one drug as initial therapy for patients with $\mathrm{BP}>20 \mathrm{mmHg}$ systolic or $>10 \mathrm{mmHg}$ diastolic above goal, with one of the drugs in the combination being a thiazide-type diuretic. ${ }^{1}$ Treatment of hypertension with angiotensin receptor blockers (ARBs) alone or in combination is considered a first line therapy in patients without compelling indications as well as for those patients with specific compelling indications, including heart failure, diabetes, and postmyocardial infarction due to their cardiovascular morbidity and mortality benefits. ${ }^{1}$ When using combination therapy, fixed-combination agents may have the potential to increase patient adherence. ${ }^{4}$ Many fixed-combination antihypertensive agents are available on the US market, and four of them are prescribed enough to be on the top 200 most prescribed drugs list in 2011.5 Azilsartan/chlorthalidone is unique in that it is the only combination drug that contains an ARB with the long-acting thiazide-like diuretic, chlorthalidone (instead of hydrochlorothiazide). It was approved on December 20, 2011 by the US Food and Drug Administration (FDA) for hypertension management. This article will discuss the clinical utility of fixed-combination azilsartan/chlorthalidone for the management of hypertension.

\section{Methods}

Peer-reviewed clinical trials, review articles, and relevant treatment guidelines, were identified from databases MEDLINE and Current Contents (both 1966 to February 15, 2013, inclusive) using search terms "azilsartan", "chlorthalidone", "pharmacology", "pharmacokinetics", "pharmacodynamics", "pharmacoeconomics", and "cost-effectiveness". The FDA website as well as manufacturer prescribing information was also reviewed to identify other relevant information..$^{9,19,25,26}$

\section{Results}

\section{Clinical pharmacology}

\section{Azilsartan}

Mechanism of action

Azilsartan selectively blocks the binding of angiotensin (AT) II to the $\mathrm{AT}_{1}$ receptors found in the vascular smooth muscle and the adrenal gland thereby promoting vasodilation and a decrease in the effects of aldosterone. ${ }^{6,7}$
Azilsartan is structurally similar to candesartan. Both azilsartan and candesartan are highly selective antagonists for the $\mathrm{AT}_{1}$ receptor compared to other $\mathrm{ARBs}$, exhibiting a greater than 10,000-fold affinity for the $\mathrm{AT}_{1}$ receptor compared to the $\mathrm{AT}_{2}$ receptor. ${ }^{8}$ Azilsartan's antihypertensive effects are not diminished by increases in renin secretion due to the its selective inhibition of the $\mathrm{AT}_{1}$ receptor. $^{7}$

\section{Pharmacokinetics}

A literature search did not reveal any pharmacokinetic studies on azilsartan published to date. However, based on materials submitted to the FDA, single and multiple dosing pharmacokinetics studies of azilsartan in the dose range of 20-320 mg have been conducted. No dosage adjustment of azilsartan is warranted based on a patient's age gender, race, or degree of renal/hepatic impairment.

Azilsartan medoxomil is a prodrug that is hydrolyzed to its active moiety, azilsartan, in the gastrointestinal tract. Peak plasma concentrations of azilsartan are reached in 1.5-3 hours following oral administration. Coadministration with food does not affect the bioavailability of azilsartan. The absolute bioavailability of azilsartan is approximately $58 \%$. $^{7}$ The volume of distribution of azilsartan is estimated to be $30 \mathrm{~L}$ in healthy subjects. Azilsartan is greater than $99 \%$ bound to plasma proteins and is concentration independent. ${ }^{7}$ Azilsartan is metabolized in the liver via cytochrome P450 (CYP) 2C9 to its inactive metabolite, M-II (formed by O-dealkylation). Azilsartan is also metabolized to a smaller extent by CYP2B6 and CYP2C8 to another inactive metabolite, M-I (formed by decarboxylation). Systemic exposure to M-II and M-I was approximately $50 \%$ and less than $1 \%$ of azilsartan, respectively. Azilsartan does not inhibit or induce the CYP system. However, azilsartan medoximil (the prodrug), and not azilsartan, is an inhibitor of the efflux transporter, p-glycoprotein. Azilsartan is primarily excreted in the urine as inactive metabolites. The renal clearance of azilsartan is $2.3 \mathrm{~mL} /$ minute. Based on radiolabeled animal studies in which a $\mathrm{C} 14$ radiolabeled dose of azilsartan was given via the oral route, approximately $97 \%$ of the administered dose was recovered in 14 days, of which $42 \%$ was recovered in urine (15\% of the dose excreted as azilsartan) and 55\% in feces. The elimination half-life of azilsartan is approximately 11 hours, with steady-state plasma concentrations reached by day five after oral administration. ${ }^{7}$

\section{Drug interactions}

A literature search did not reveal any drug interaction studies on azilsartan published to date. Azilsartan is 
metabolized by CYP2C9. Based on data submitted to the FDA, drug interactions have been studied with azilsartan and caffeine, antacid, warfarin, digoxin, tolbutamide, glyburide, metformin, pioglitazone, chlorthalidone, amlodipine, dextromethorphan, midazolam, and fexofenandine. No significant interactions were observed between these agents and azilsartan.

In another drug interaction study of 36 healthy volunteers with an average age of 30 years, subjects were given azilsartan $40 \mathrm{mg}$ daily for 1 day, fluconazole $200 \mathrm{mg}$ daily or ketoconazole $400 \mathrm{mg}$ daily for 5 days, and azilsartan $40 \mathrm{mg}$ plus fluconazole $200 \mathrm{mg}$ daily or ketoconazole $400 \mathrm{mg}$ for 1-day for three consecutive periods with a 3-day washout inbetween. Clearance of azilsartan was reduced when coadministered with fluconazole (CYP2C9 inhibitor) with an increase in area under the curve by $\sim 40 \%$. When coadministered with ketoconazole (a CYP3A4/5 inhibitor), the area under the curve of azilsartan was decreased by approximately $20 \%$. The clinical significance of this interaction was not examined.

Although not specifically evaluated, the manufacturer Novartis (East Hanover, NJ, USA) has issued a warning in their prescribing information cautioning the use of azilsartan with non-steroidal anti-inflammatory agents and COX-2 inhibitors. ${ }^{7}$ Angiotensin II increases the glomerular filtration rate by causing constriction of the efferent arteriole. Decreased angiotensin II binding to the receptor with azilsartan use decreases the glomerular filtration rate because of dilatation of the efferent arteriole. Non-steroidal anti-inflammatory agents and COX-2 inhibitors cause prerenal acute renal failure by blocking prostaglandin production, which also alters local glomerular arteriolar perfusion. ${ }^{9}$ Concurrent use of these agents may result in additive risk in deterioration of renal function.

\section{Compared to other ARBs}

The antihypertensive effects of azilsartan has been compared to olmesartan and valsartan. The effect of azilsartan on cardiovascular events/outcomes has not been studied.

Bakris et al performed a randomized, placebo-controlled study, comparing the antihypertensive efficacy and safety of azilsartan, olmesartan, and placebo. ${ }^{10}$ One thousand two hundred and seventy-five patients aged over 18 years of age, with primary hypertension (systolic blood pressure [SBP] between 150-180 $\mathrm{mmHg}$ ) were randomized to placebo, or azilsartan $20 \mathrm{mg}, 40 \mathrm{mg}$, or $80 \mathrm{mg}$ daily, or olmesartan $40 \mathrm{mg}$ daily for 6 weeks. Dose-dependent reductions in 24-hour mean SBP (measured by 24 hours ambulatory blood pressure monitoring $[\mathrm{ABPM}])$ at study end occurred in all azilsartan groups $(20 \mathrm{mg},-12.2 \mathrm{mmHg} ; 40 \mathrm{mg}$, $-13.5 \mathrm{mmHg}$; $80 \mathrm{mg},-14.6 \mathrm{mmHg}$ ) and olmesartan $40 \mathrm{mg}$ group $(-12.6 \mathrm{mmHg}), P<0.05$ for all. Reduction in 24-hour mean SBP was greater with azilsartan $80 \mathrm{mg}$ than olmesartan $40 \mathrm{mg}$ by $2.1 \mathrm{mmHg}$ (95\% confidence interval [CI] -4.0 to $-0.1 ; P=0.038$ ), while azilsartan $40 \mathrm{mg}$ was not inferior to olmesartan $40 \mathrm{mg}$. The side effect profiles of both angiotensin receptor blockers were similar to placebo (not statistically compared). The study demonstrated that azilsartan is well tolerated and more efficacious at its maximal dose than the highest recommended dose of olmesartan in this 6-week study.

White et al performed a double-blind, randomized, placebo-controlled trial comparing the antihypertensive efficacy and safety of azilsartan, olmesartan, valsartan, and placebo. ${ }^{11}$ One thousand two hundred and eighty-five patients aged over 18 years of age with primary hypertension (SBP between $150-180 \mathrm{mmHg}$ ) were randomized to placebo, or azilsartan 40-80 mg daily, olmesartan $40 \mathrm{mg}$ daily, or valsartan $320 \mathrm{mg}$ daily for 6 weeks. The mean age of participants was 56 years. Azilsartan at $80 \mathrm{mg}$ had superior efficacy to both valsartan at $320 \mathrm{mg}$ and olmesartan at $40 \mathrm{mg}$ : placebo-adjusted 24-hour SBP (measured by ABPM) was lowered $(-14.3 \mathrm{mmHg})$ more than $320 \mathrm{mg}$ of valsartan $(-10.0 \mathrm{mmHg} ; P<0.001$ azilsartan versus valsartan $)$ and $40 \mathrm{mg}$ of olmesartan $(-11.7 \mathrm{mmHg} ; P<0.009$ azilsartan versus olmesartan). Azilsartan at $40 \mathrm{mg}$ was not inferior to $40 \mathrm{mg}$ of olmesartan (difference: $-1.4 \mathrm{mmHg}$ [95\% CI: -3.3 to 0.5$]$ ). Safety and tolerability were similar among the placebo and four active treatments (not statistically compared). This study demonstrated that azilsartan at its maximum dose is more efficacious than both olmesartan and valsartan at their maximum approved doses without increasing adverse events.

Sica et al performed a double-blind, randomized trial comparing the antihypertensive efficacy and safety of azilsartan and valsartan. ${ }^{12}$ Nine hundred and eighty-four patients aged over 18 years of age with primary hypertension (SBP between 150-180 $\mathrm{mmHg}$ ) were randomized to placebo, or azilsartan $20 \mathrm{mg}$ titrated to $40 \mathrm{mg}, 40 \mathrm{mg}$ titrated to $80 \mathrm{mg}$ daily, or valsartan $80 \mathrm{mg}$ titrated to $320 \mathrm{mg}$ for 24 weeks. The mean age of participants was 58 years. Azilsartan $40 \mathrm{mg}$ and $80 \mathrm{mg}$ lowered 24-hour mean SBP $(-14.9 \mathrm{mmHg}$ and $-15.3 \mathrm{mmHg}$, respectively) more than valsartan $320 \mathrm{mg}$ $(-11.3 \mathrm{mmHg} ; P<0.001$ for $40 \mathrm{mg}$ and $80 \mathrm{mg}$ comparisons versus valsartan). This study demonstrated that azilsartan across the effective dose range is more effective than 
valsartan at its maximum recommended dose for lowering BP with a similar adverse event profile.

\section{Chlorthalidone}

\section{Mechanism of action}

Though commonly considered a thiazide, unlike hydrochlorothiazide, chlorthalidone is not a benzothiadiazine and is more appropriately called a thiazide-like diuretic. ${ }^{13}$ Although structurally different, chlorthalidone and thiazide diuretics appear to produce their diuretic effect by direct effect on the distal convoluted tubule of the nephron, resulting in decreased extracellular fluid volume, plasma volume, and total exchangeable sodium. It is postulated that sodium and water depletion provide a basis for its antihypertensive effect.

\section{Pharmacokinetics}

Chlorthalidone is $100 \%$ absorbed with serum concentrations peak at approximately $2-6$ hours after oral administration. ${ }^{14,15}$ One randomized, crossover study in healthy volunteers found that peak serum concentrations after single doses of $50 \mathrm{mg}, 100 \mathrm{mg}$, or $200 \mathrm{mg}$ were $3.2 \mu \mathrm{g} / \mathrm{mL}, 5.6 \mu \mathrm{g} / \mathrm{mL}$, and $7.9 \mu \mathrm{g} / \mathrm{mL}$, respectively. ${ }^{14}$ The half-life of chlorthalidone in this single-dose study was approximately 42 hours (range, 29-55 hours, longer than hydrochlorothiazide, which ranged from 6-9 hours). Interindividual variability in the half-life of chlorthalidone is large, with typical mean half-lives of 45 to 60 hours after long-term dosing. ${ }^{14,15}$ Chlorthalidone serum concentrations after $100 \mathrm{mg}$ are only twice those of a $25 \mathrm{mg}$ dose, indicating a flat dose-serum concentration curve. ${ }^{14}$ Chlorthalidone rapidly enters (half-life, 15 minutes) and concentrates in erythrocytes. ${ }^{16}$ Studies have found 7 to 10 times greater concentrations of chlorthalidone in erythrocytes than in plasma. ${ }^{14,15}$ One possible explanation for the long half-life is that chlorthalidone concentrates in erythrocytes and is slowly released from this compartment. The natriuretic effect of chlorthalidone was maximal at 18 hours and lasted greater than 48 hours. Although the half-life of chlorthalidone is relatively long, one double-blind study found that three times weekly dosing of chlorthalidone did not completely control SBP, suggesting that every-other-day dosing might not be maximally effective. ${ }^{17}$ The major portion of the drug is excreted unchanged by the kidneys. Nonrenal routes of elimination have yet to be clarified. Data are not available regarding percentage of dose as unchanged drug and metabolites, concentration of the drug in body fluids, degree of uptake by a particular organ or in the fetus, or passage across the blood-brain barrier. ${ }^{18}$

\section{Drug interactions}

No significant direct drug interactions with chlorthalidone have been reported. If chlorthalidone causes significant hypokalemia, the risk of arrhythmia may increase when used with other medications that have the risk to prolong QT interval. Lithium renal clearance is reduced by diuretics, such as chlorthalidone, increasing the risk of lithium toxicity. ${ }^{18}$

\section{Compared to thiazides}

The antihypertensive effects of chlorthalidone and hydrochlorothiazide have been compared. ${ }^{13}$ The earliest study of dose equivalence was a randomized, doubleblind study of placebo, hydrochlorothiazide $100 \mathrm{mg}$ daily, and chlorthalidone $50 \mathrm{mg}$ daily, each given for 6 weeks separated by a 2 -week washout. Pretreatment BPs were $\leq 180 / 110 \mathrm{mmHg}$. Chlorthalidone $50 \mathrm{mg}$ reduced BP by $25 / 10 \mathrm{mmHg}$, and hydrochlorothiazide $100 \mathrm{mg}$ reduced $\mathrm{BP}$ by $18 / 8 \mathrm{mmHg}$ (no significant difference between the two agents). Another double-blind, randomized study evaluated of hydrochlorothiazide $50 \mathrm{mg}$ twice daily (100 mg daily) compared to chlorthalidone $50 \mathrm{mg}$ daily. After 4 weeks of treatment, chlorthalidone reduced mean BP by $18 / 15 \mathrm{mmHg}$, whereas hydrochlorothiazide reduced mean BP by $22 / 16 \mathrm{mmHg}$ (no significant difference between drugs). The percentage of patients with diastolic BP $<90 \mathrm{mmHg}$ was $42 \%$ with chlorthalidone and $32 \%$ with hydrochlorothiazide.

Although no prospective, randomized trials assessing cardiovascular outcomes between chlorthalidone and hydrochlorothiazide have been conducted, evidence indicates that chlorthalidone may be superior to hydrochlorothiazide. ${ }^{19}$ The Multiple Risk Factor Intervention Trial (MRFIT) was first to propose a clinical benefit with chlorthalidone compared to hydrochlorothiazide. ${ }^{19}$ MRFIT was a large randomized primary prevention trial assessing the efficacy of a multifactor intervention program, which included dietary advice, smoking cessation counseling, and hypertension treatment, compared to usual care. Patients in the study could receive chlorthalidone or hydrochlorothiazide for hypertensive therapy. Approximately 5 years after randomization, the diuretic treatment protocol was changed to replace treatment with hydrochlorothiazide to chlorthalidone due to a $44.1 \%$ higher coronary heart disease mortality in clinics predominantly prescribing hydrochlorothiazide compared to the usual care group $(P=0.23)$, whereas the clinics predominantly prescribing chlorthalidone had a $-58.2 \%$ (reduction) in coronary heart disease mortality compared to usual care. After the change was instituted, the 
rate of coronary heart disease mortality in the previously predominant hydrochlorothiazide clinics decreased to $-7.9 \%$ when compared to usual care. A subsequent retrospective cohort analysis of the data from MRFIT demonstrated that cardiovascular events were significantly lower in those on chlorthalidone (adjusted hazard ratio 0.51 [95\% CI 0.43-0.62], $P<0.0001)$ and on hydrochlorothiazide (adjusted hazard ratio 0.65 [95\% CI 0.55-0.75], $P<0.0001$ ) compared to those who took neither drug. When comparing the two drugs, chlorthalidone had significantly fewer cardiovascular events compared to hydrochlorothizide $(P=0.0016)$. Patients receiving chlorthalidone also had significantly lower SBP $(P<0.0001)$, lower low-density lipoprotein $(P=0.0009)$, lower potassium $(P=0.0003)$, and higher uric acid $(P<0.0001)$ over time compared to hydrochlorothiazide. ${ }^{20}$

\section{Clinical trials}

Azilsartan plus chlorthalidone combination has been evaluated for hypertension management. A dose response study, a study comparing the efficacy of azilsartan plus chlorthalidone and azilsartan plus hydrochlorothiazide, and a study comparing the efficacy of azilsartan plus chlorthalidone and olmesartan plus hydrochlorothiazide have been published in the literature to date. ${ }^{21-23}$ In addition, based on materials submitted to the FDA, two other efficacy studies have also been performed (Table 1). ${ }^{23}$ The studies evaluating the efficacy of azilsartan plus chlorthalidone for management of hypertension will be reviewed below.

Sica et al performed a study comparing the efficacy and safety of fixed-dose combinations of the azilsartan and chlorthalidone with the individual monotherapies in a double-blind factorial study. ${ }^{21} \mathrm{~A}$ total of 1714 patients age $>18$ years, with SBP $160 \mathrm{mmHg}$ to $190 \mathrm{mmHg}$ inclusive were randomized to azilsartan $0 \mathrm{mg}, 20 \mathrm{mg}$, $40 \mathrm{mg}$, or $80 \mathrm{mg}$ and /or chlorthalidone $0 \mathrm{mg}, 12.5 \mathrm{mg}$, or $25 \mathrm{mg}$. The primary efficacy end point was change of mean SBP (measured by 24 hour ABPM) from baseline to 8 weeks. Baseline BP was an averaged $165 / 95 \mathrm{mmHg}$. For the pooled azilsartan/chlorthalidone $40 / 25 \mathrm{mg}$ and $80 / 25 \mathrm{mg}$ fixed dose combination groups, SBP reduction was $28.9 \mathrm{mmHg}$ (similar between $40 / 25 \mathrm{mg}$ and $80 / 25 \mathrm{mg}$ ) and exceeded azilsartan $80 \mathrm{mg}$ and chlorthalidone $25 \mathrm{mg}$ monotherapies by $13.8 \mathrm{mmHg}$ and $13 \mathrm{mmHg}$, respectively ( $P<0.001$ for both comparisons). Each of the six individual azilsartan/chlorthalidone doses led to significantly greater reductions in SBP compared to their respective azilsartan and chlorthalidone components. Treatment with azilsartan/ chlorthalidone resulted in a similar magnitude of BP reduction in African American patients compared with the overall population, even though African American patients have been previously demonstrated to be less responsive to azilsartan alone. ${ }^{24}$ Discontinuation rates and elevations in serum creatinine were dose-dependent and occurred more often in the combination azilsartan/chlorthalidone groups ( $0.6 \%-5 \%$ compared to $<1 \%$ in monotherapy groups). In patients with stage two hypertension, the investigators concluded that, as expected, treatment with the combination of azilsartan and chlorthalidone resulted in substantially greater SBP reduction compared with either agent alone.

Cushman et al compared the efficacy of once-daily fixeddose combinations of azilsartan/chlorthalidone force titrated to a high dose of either $40 / 25 \mathrm{mg}$ or $80 / 25 \mathrm{mg}$ with a fixeddose combination of the olmesartan/hydrochlorothiazide force titrated to $40 / 25 \mathrm{mg} .{ }^{22}$ One thousand and seventyone subjects aged $>18$ years, with baseline SBP between 160 to $190 \mathrm{mmHg}$ and diastolic $\mathrm{BP} \leq 119 \mathrm{mmHg}$ were randomized. The primary efficacy end point was change of mean SBP (measured by 24 hours ABPM) from baseline to 12 weeks. Changes in SBP at week 12 were significantly greater in both azilsartan/chlorthalidone arms than in the olmesartan/hydrochlorothiazide arm (42.5 $\mathrm{mmHg}, 44.5 \mathrm{mmHg}$ and $37.1 \mathrm{mmHg}$ respectively, $P<0.001$ for all comparisons). Adverse events leading to permanent drug discontinuation occurred in $7.9 \%, 14.5 \%$, and $7.1 \%$ of the groups given azilsartan/chlorthalidone $40 / 25 \mathrm{mg}$, azilsartan/chlorthalidone $80 / 25 \mathrm{mg}$, and olmesartan/hydrochlorothiazide $40 / 25 \mathrm{mg}$, respectively. The investigators concluded that azilsartan/chlorthalidone combination was more effective in reducing BP than olmesartan/hydrochlorothiazide combination. It is, however, important to note that the approximate equivalent dose of chlorthalidone $25 \mathrm{mg}$ is hydrochlorothiazide $50 \mathrm{mg}$. Therefore, it is possible that the unequal potency doses were being compared.

Bakris et al compared the efficacy of azilsartan/ chlorthalidone combination and azilsartan/hydrochlorothiazide combination. ${ }^{23}$ Six hundreds and nine patients aged $>18$ years with stage 2 primary hypertension were randomized to receive $12.5 \mathrm{mg}$ of chlorthalidone or hydrochlorothiazide in addition to azilsartan for 4 weeks, and titrated up to $25 \mathrm{mg}$ for another 4 weeks if BP was not controlled, after receiving azilsartan $40 \mathrm{mg}$ alone for 2 weeks. Target BP was defined as clinical BP $<140 / 90 \mathrm{mmHg}$ for participants without diabetes or chronic kidney disease and $<130 / 80 \mathrm{mmHg}$ for participants with diabetes or chronic kidney disease. 
Table I Pertinent clinical studies of azilsartan/chlorthalidone in hypertension management

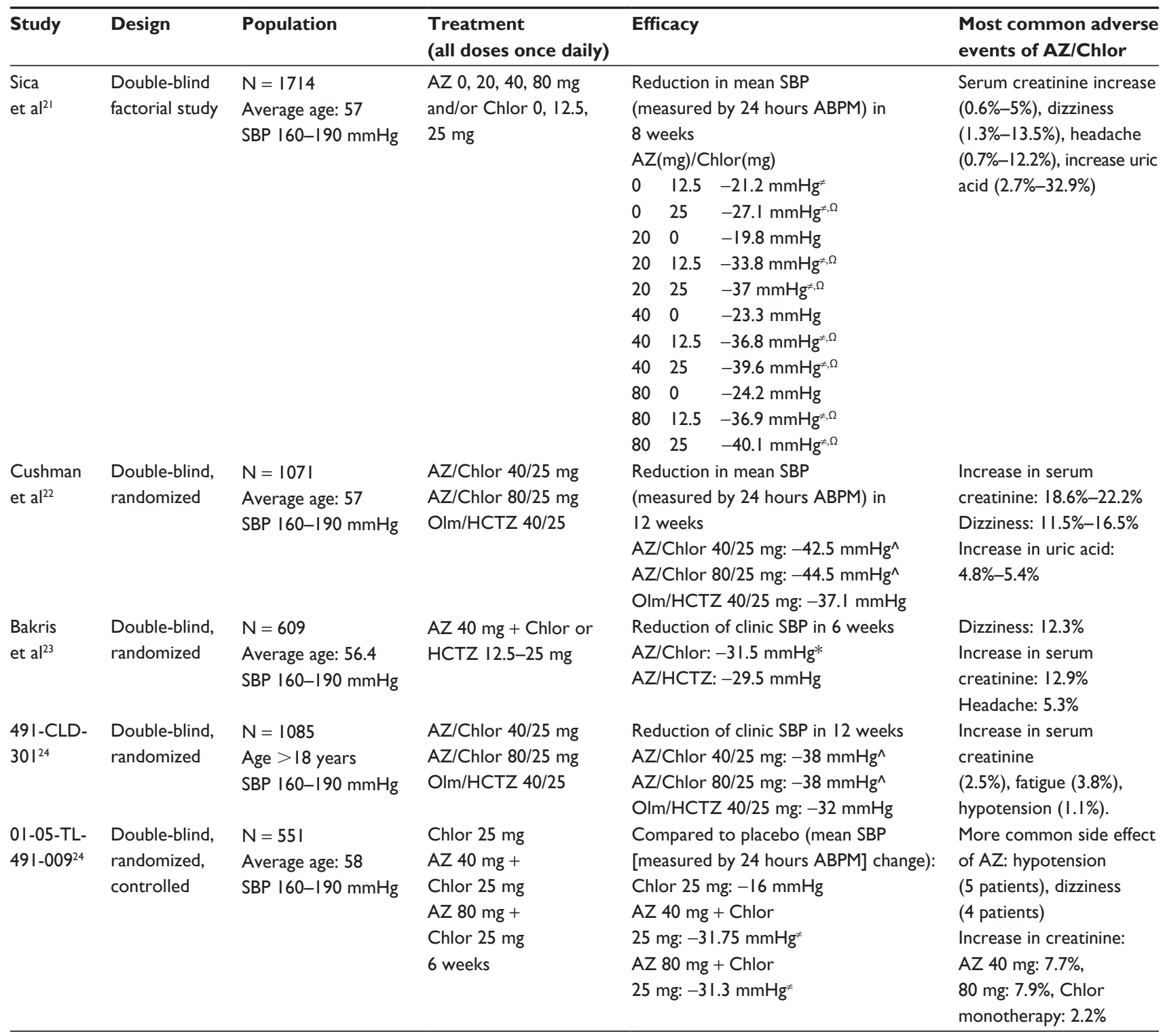

Notes: $* P<0.05$ compared to azilsartan/HCTZ; $\wedge p<0.05$ compared to olmesartan/HCTZ; $\neq p<0.05$ compared to chlorthalidone alone; $\Omega$ P $<0.05$ compared to azilsartan alone.

Abbreviations: ABPM, ambulatory blood pressure monitoring; AZ, azilsartan; Chlor, chlorthalidone; Olm, olmesartan; HCTZ, hydrochlorothiazide; SBP, systolic blood pressure.

Azilsartan/chlorthalidone combinations provided greater reduction in SBP than azilsartan/hydrochlorothiazide combinations $(31.5 \mathrm{mmHg}$ versus $29.5 \mathrm{mmHg}, P<0.001$ ). The percentage of participants achieving target BP at week 6 was greater for the chlorthalidone versus hydrochlorothiazide combination (64.1\% versus $45.9 \%, P<0.001$ ). Drug discontinuations due to adverse events were not statistically significantly different between groups $(9.3 \%$ versus $7.3 \%$, $P=0.38$ ), and hypokalemia was uncommon in both groups. The investigators concluded that chlorthalidone combined with azilsartan provides better BP control than hydrochlorothiazide combined with azilsartan. Similar to the Cushman et al study discussed above, it is important to note that the approximate equivalent dose of chlorthalidone $25 \mathrm{mg}$ is hydrochlorothiazide $50 \mathrm{mg}$. Therefore, it is possible that the unequal potency doses were being compared.

Study 491-CLD-301 compared the efficacy of once-daily fixed-dose combinations of azilsartan/chlorthalidone of $20 / 12.5 \mathrm{mg}$, or 40/25 mg with a fixed-dose combination and olmesartan/hydrochlorothiazide $20 / 12.5 \mathrm{mg} .{ }^{25}$ One thousand and eighty-five subjects aged $>18$ years, with baseline SBP between 160 to $190 \mathrm{mmHg}$ and diastolic $\mathrm{BP} \leq 119 \mathrm{mmHg}$ 
were randomized. If subjects achieved both target SBP and diastolic BP $(<140 / 90 \mathrm{mmHg}$ for subjects without diabetes or chronic kidney diseases or $<130 / 80 \mathrm{mmHg}$ for subjects with diabetes or chronic kidney disease) by the end of Week 4, they continued to receive their starting dose for the duration of the study. For subjects who did not achieve both target SBP and diastolic BP by Week 4, study drug was titrated to azilsartan/ chlorthalidone 40/25 mg, 80/25 mg, and olmesartan/ hydrochlorothiazide $40 / 25 \mathrm{mg}$. The primary endpoint was the change from baseline to Week 8 in SBP. SBP reductions at week 8 in both azilsartan/chlorthalidone treatment groups (33 to $38 \mathrm{mmHg}$ ) were significantly $(P<0.05)$ greater than in the olmesartan/hydrochlorothiazide group (27 to $32 \mathrm{mmHg}$ ). Approximately $13 \%$ of subjects prematurely discontinued the study, including $15 \%$ and $14 \%$ in the azilsartan/chlorthalidone low-dose and high-dose groups, respectively, and $9 \%$ in the olmesartan/hydrochlorothiazide group. The most common side effects in the azilsartan/chlorthalidone group included serum creatinine elevation ( $2.5 \%$ in the high dose group), fatigue (3.8\% in the high dose group), and hypotension ( $1.1 \%$ in the high dose group). It is again important to note that the approximate equivalent dose of chlorthalidone $25 \mathrm{mg}$ is hydrochlorothiazide $50 \mathrm{mg}$. Therefore, it is possible that the unequal potency doses were being compared.

Study 01-05-TL-491-009 is a double-blind, randomized, placebo-controlled study to evaluate the efficacy and safety of azilsartan when coadministered with chlorthalidone. ${ }^{25}$ Five hundred and fifty-one subjects with SBP 160-190 mmHg and aged $>18$ years were randomized to received placebo plus chlorthalidone $25 \mathrm{mg}$ daily, azilsartan $40 \mathrm{mg}$ daily plus chlorthalidone $25 \mathrm{mg}$ daily, or azilsartan $80 \mathrm{mg}$ plus chlorthalidone $25 \mathrm{mg}$ daily for 6 weeks. At the end of the treatment period, chlorthalidone alone decreased mean SBP (measured by 24 hours ABPM) by $16 \mathrm{mmHg}$. There was no differences between the two azilsartan and chlorthalidone groups (40 mg, $-31.72 \mathrm{mmHg}$; $80 \mathrm{mg},-31.3 \mathrm{mmHg}$; $P>0.05)$ but both groups compared to chlorthalidone alone significantly reduced SBP $(P<0.05$ for both). Adverse events of hypotension (five patients), dizziness (four patients), and asthenia (one patient) were reported in the azilsartan groups. However, the increases in serum creatinine ( $\geq 30 \%$ from baseline) from baseline in the azilsartan $40 \mathrm{mg}$ and $80 \mathrm{mg}$ combination groups were more than double the increases reported in the chlorthalidone monotherapy group (increase in creatinine-azilsartan $40 \mathrm{mg}, 28$ patients $(7.7 \%)$, and $80 \mathrm{mg}$, 41 patients $(7.9 \%)$; chlorthalidone monotherapy: 4 patients (2.2\%), $P$-value not reported). This study demonstrated that azilsartan, when used with chlorthalidone, produced a further reduction in BP. However, creatinine needs to be monitored for potential worsening of renal function.

Overall, azilsartan/chlorthalidone has demonstrated the ability to lower BP in numerous randomized, double blind, placebo controlled trials. It appears that there is little difference in BP lowering effect among the azilsartan doses of 40 and $80 \mathrm{mg}$. The BP lowering effect appeared to be superior to olmesartan and hydrochlorothiazide.

\section{Adverse events}

According to the manufacturer (Novartis), a total of 3900 patients were evaluated in premarketing clinical trials for safety when treated with azilsartan/chlorthalidone for 6 months to 1 -year. ${ }^{18}$ Azilsartan/chlorthalidone was generally well tolerated and adverse reactions have generally been mild and transient in nature. Common adverse reactions that occurred in the 8-week factorial design trial included dizziness $(8.9 \%)$ and fatigue $(2 \%) .{ }^{18}$ Hypotension and syncope were reported in $1.7 \%$ and $0.3 \%$, respectively, of patients treated with azilsartan/chlorthalidone. Study discontinuation because of adverse reactions occurred in $8.3 \%$ of patients treated with the recommended doses of azilsartan/chlorthalidone combination compared to $3.2 \%$ of patients treated with azilsartan and $3.2 \%$ of patients treated with chlorthalidone. The most common reasons for discontinuation of therapy with azilsartan/chlorthalidone were serum creatinine increase (3.6\%) and dizziness $(2.3 \%)$. The adverse reaction profile obtained from 52 weeks of open-label combination therapy with azilsartan plus chlorthalidone was similar to that observed during the double-blind, active controlled trials, with an overall incidence of adverse reactions similar to placebo. Increased serum creatinine is a known pharmacologic effect of renin-angiotensin aldosterone system blockers, such as ARBs and angiotensin converting enzyme (ACE) inhibitors, and is related to the magnitude of $\mathrm{BP}$ reduction. The incidence of consecutive increases of creatinine $\geq 50 \%$ from baseline was $2.0 \%$ in patients treated with the recommended doses of azilsartan/chlorthalidone compared to $0.4 \%$ and $0.3 \%$ with azilsartan and chlorthalidone alone, respectively. Elevations of creatinine were typically transient, or nonprogressive and reversible. Mean increases in blood urea nitrogen were observed with azilsartan/chlorthalidone $(5.3 \mathrm{mg} / \mathrm{dL})$ compared to azilsartan $(1.5 \mathrm{mg} / \mathrm{dL})$ and with chlorthalidone (2.5 mg/dL).

\section{Contraindications/precautions}

According to the manufacturer (Novartis), azilsartan/ chlorthalidone is contraindicated in patients with anuria. ${ }^{18}$ 
The manufacturer cautions against the use of azilsartan/ chlorthalidone in pregnant woman, as drugs that act directly on the renin-angiotensin system can cause fetal and neonatal morbidity and death when administered to pregnant women during the second and the third trimester. ${ }^{18}$ Azilsartan/ chlorthalidone is pregnancy category D. ${ }^{18}$ In addition, patients who are volume- or salt-depleted may be more sensitive to the hypotension effect of azilsartan. In patients whose renal function may depend on the activity of the renin-angiotensin system (including severe congestive heart failure, renal artery stenosis, or volume depletion), treatment with ACE inhibitors and ARBs has been associated with oliguria or progressive azotemia. Hypokalemia is a dose-dependent adverse reaction that may develop with chlorthalidone. Coadministration of digitalis may exacerbate the adverse effects of hypokalemia. Azilsartan may attenuate chlorthalidone-associated hypokalemia. Hyperuricemia may occur or gout may be precipitated in some patients receiving chlorthalidone or thiazide diuretics.

\section{Dosage and administration}

The recommended starting dose of azilsartan/chlorthalidone is $40 / 12.5 \mathrm{mg}$ taken orally once daily. Most of the antihypertensive effect is apparent within 1 to 2 weeks. The dosage may be increased to $40 / 25 \mathrm{mg}$ after 2 to 4 weeks as needed to achieve BP goals. Azilsartan/chlorthalidone doses above $40 / 25 \mathrm{mg}$ probably do not provide significant additional antihypertensive effects.

\section{Pharmacoeconomics/cost-effectiveness}

A literature search reviewed no formal cost-effectiveness study performed for azilsartan to date. The average wholesale price of azilsartan/chlorthalidone in the US is $\$ 109.60$ for a 30-day supply for both the 40/12.5 $\mathrm{mg}$ and the $40 / 25 \mathrm{mg}$ tablets. ${ }^{26}$ In terms of acquisition cost, azilsartan/ chlorthalidone is less expensive than other ARB/thiazide combinations on the market (Table 2).

\section{Discussion/future perspective}

Clinical studies to date demonstrated that the azilsartan/ chlorthalidone combination is effective in lowering BP and more effective than either single agent alone. Its antihypertensive effect also appears to be better than the olmesartan/hydrochlorothiazide combination. Medications that have direct effects on the renin-angiotensin aldosterone system, such as ACE inhibitors, ARBs, and aldosterone antagonists have been found to not only be effective in lowering BP, but also improve mortality and morbidity
Table 2 Comparative cost (average wholesale price) of angiotensin receptor blocker-diuretic combination used in the United States ${ }^{24}$

\begin{tabular}{ll}
\hline Antihypertensives & $\begin{array}{l}\text { Cost for 30-day } \\
\text { supply (US } \$ \text { ) }\end{array}$ \\
\hline Azilsartan/chlorthalidone tablet & 109.60 \\
$40-12.5 \mathrm{mg}$ & 109.60 \\
$40-25 \mathrm{mg}$ & \\
Candesartan/hydrochlorothiazide tablet & 368.18 \\
16-12.5 mg & 375.51 \\
$32-12.5 \mathrm{mg}$ & 406.48 \\
$32-25 \mathrm{mg}$ & \\
Olmesartan/hydrochlorothiazide tablet & 119.88 \\
$20-12.5 \mathrm{mg}$ & 167.04 \\
$40-12.5 \mathrm{mg}$ & 501.12 \\
$40-25 \mathrm{mg}$ & \\
Valsartan/hydrochlorothiazide tablet & 117.99 \\
$80-12.5 \mathrm{mg}$ & 128.37 \\
$160-12.5 \mathrm{mg}$ & 145.58 \\
$160-25 \mathrm{mg}$ & 162.44 \\
$320-12.5 \mathrm{mg}$ & 184.29 \\
$320-25 \mathrm{mg}$ & \\
Losartan/hydrochlorothiazide tablet & 75.05 \\
$50-12.5 \mathrm{mg}$ & 102.23 \\
100-25 mg & \\
Telmisartan/hydrochlorothiazide tablet & 133.96 \\
$40-12.5 \mathrm{mg}$ & 133.96 \\
$80-25 \mathrm{mg}$ & 488.15 \\
Eprosartan/hydrochlorothiazide tablet & 488.15 \\
$600-12.5 \mathrm{mg}$ & \\
$600-25 \mathrm{mg}$ & \\
\hline
\end{tabular}

in patients with heart failure (ACE inhibitors, $\mathrm{ARBs}$, and aldosterone antagonists), history of myocardial infarction (ACE inhibitors, ARBs, and aldosterone antagonists), and nephropathy (ACE inhibitors and ARBs). Whether azilsartan will be as effective of other ARBs (valsartan, candesartan, irbesartan) is not known. A literature review did not identify any published studies performed evaluating the effect of azilsartan on long-term vascular outcomes. Further research investigating these potential roles will help establish a place for azilsartan in cardiovascular disease management, in addition to hypertension treatment. Chlorthalidone has been demonstrated to improve cardiovascular outcomes.

The current JNC7 guidelines recommend administering more than one drug as initial antihypertensive therapy for patients with BP $>20 \mathrm{mmHg}$ systolic or $>10 \mathrm{mmHg}$ diastolic above goal, with one of these drugs being a thiazide and thiazide-like diuretic. ${ }^{1}$ As seen in the Antihypertensive and Lipid-Lowering treatment to prevent Heart Attack Trial (ALLHAT), $63 \%$ of the study population was eventually on two or more antihypertensive agents and at 
the end of 5 years only $66 \%$ had achieved controlled BP. ${ }^{28}$ Numerous newer trials have also shown high rates of patients requiring more than one drug for BP management, such as INternational VErapamil SR trandolapril STudy (INVEST) (80\%), Anglo-Scandinavian Cardiac Outcomes Trial: Blood Pressure-Lowering Limb (ASCOT-BPLA) (78\%), and Losartan Intervention For Endpoint (LIFE) (88\%). ${ }^{28-30}$ Since a large number of hypertensive patients require more than one drug to control their hypertension, many trials have examined the benefit of combinations of antihypertensive medications as well as the benefit of combination therapy as initial treatment. The prompter achievement of BP goal may also be a benefit of initial combination therapy opposed to initial monotherapy later progressing to combination therapy. Results from the Valsartan Antihypertensive Long-term Use Evaluation (VALUE) trial, comparing a valsartan-based BP regimen to an amlodipine-based $\mathrm{BP}$ regimen, suggest that obtaining BP control within the first month of treatment is correlated to a lower rate of cardiovascular events. ${ }^{31} \mathrm{~A}$ large, case-control study showed an $11 \%$ decrease in cardiovascular events when patients receiving antihypertensive medications were initiated on combination therapy instead of monotherapy (odds ratio OR 0.74; 95\% CI: $0.65-0.85$ ). ${ }^{32}$ These data suggest that the best outcomes may be achieved when combination therapy is initially started in hypertensive patients. The combination of azilsartan/chlorthalidone has not been tested in specific study designs.

Currently, azilsartan/chlorthalidone can be considered an alternative antihypertensive regimen for patients who require combination therapy. Serum creatinine needs to be monitored when patients are receiving combination therapy as it has been demonstrated in clinical studies that the incidence of elevation of serum creatinine in combination therapy is higher than using individual agents alone.

\section{Conclusion}

Azilsartan is a new ARB with high affinity to the $\mathrm{AT}_{1}$ receptor that is approved by the FDA for hypertension management. Unlike other ARBs, azilsartan has no clinical data supporting improvement of cardiovascular outcomes, and is not approved for indications which selected other ARBs may be used for (eg, diabetic nephropathy and heart failure). Chlorthalidone is a longer acting thiazide like diuretic that has been demonstrated to improve cardiovascular outcomes. Compared to olmesartan/hydrochlorothiazide and azilsartan/ hydrochlorothiazide combinations, azilsartan/chlorthalidone appears to be more efficacious in reducing BP. Azilsartan/chlorthalidone can be considered as an antihypertensive therapy option in patients whom combination therapy is required (BP $>20 \mathrm{mmHg}$ systolic or $>10 \mathrm{mmHg}$ diastolic above goal). Cost to patients and insurance coverage will probably determine whether azilsartan/chlorthalidone would be the most appropriate combination therapy for an individual patient.

\section{Disclosure}

The author declares no conflicts of interest in this work.

\section{References}

1. National Institutes of Health; National Heart, Lung, and Blood Institute. The Seventh Report of the Joint National Committee on Prevention, Detection, Evaluation and Treatment of High Blood Pressure. Bethesda, MD, USA: US Department of Health and Human Services; 2004. Available from: http://www.nhlbi.nih.gov/guidelines/hypertension/ jnc7full.pdf. Accessed on August 24, 2011.

2. Lloyd-Jones D, Adams RJ, Brown TM, et al; American Heart Association Statistics Committee and Stroke Statistics Subcommittee. Executive summary: heart disease and stroke statistics - 2010 update: a report from the American Heart Association. Circulation. 2010;121(7):948-954.

3. http://www.cdc.gov [homepage on the Internet]. High Blood Pressure Facts. Centers for Disease Control and Prevention; 2011. Available from: http://www.cdc.gov/bloodpressure/facts.htm. Accessed July 24, 2011.

4. Gupta AK, Arshad S, Poulter NR. Compliance, safety, and effectiveness of fixed-dose combinations of antihypertensive agents: a meta-analysis. Hypertension. 2010;55(2):399-407.

5. Pharmacy Times. Top 200 Products of 2011 by Total Prescriptions. Plainsboro, NJ, USA: Intellisphere LLC; 2012. Available from: http:// www.pharmacytimes.com/_media/_pdf/Top_200_Drugs_2011_Total_ Rx.pdf. Accessed December 20, 2012.

6. Appendix to Clinical Pharmacology Review: Edarbi ${ }^{\circledR}$. Center for Drug Evaluation and Research. Clinical Pharmacology and Biopharmaceutics Review(s). Silver Spring, MD, USA: Food and Drug Administration, 2011. Available from: http://www.accessdata.fda.gov/drugsatfda_docs/n da/2011/200796Orig1s000ClinPharmR.pdf. Accessed July 26, 2011.

7. Takeda Pharmaceuticals USA, Inc. Prescribing Information: Edarbi. Deerfield: Takeda Pharmaceuticals America, Inc; 2012.Available from: http://www.edarbi.com/. Accessed July 7, 2011.

8. Ojima $\mathrm{M}$, Igata $\mathrm{H}$, Tanaka $\mathrm{M}$, et al. In vitro antagonistic properties of a new angiotensin type 1 receptor blocker, azilsartan, in receptor binding and function studies. J Pharmacol Exp Ther. 2011;336(3):801-808.

9. Agrawal M, Swartz R. Acute renal failure. Am Fam Physician. 2000; 61(7):2077-2088.

10. Bakris GL, Sica D, Weber M, et al. The comparative effects of azilsartan medoxomil and olmesartan on ambulatory and clinical blood pressure. J Clin Hypertens (Greenwich). 2011;13(2):81-88.

11. White WB, Weber MA, Sica D, et al. Effects of angiotensin receptor blocker azilsartan medoxomil versus olmesartan and valsartan on ambulatory and clinic blood pressure in patients with stage 1 and 2 hypertension. Hypertension. 2011;57(3):413-420.

12. Sica D, White WB, Weber MA, et al. Comparison of the novel angiotensin II receptor blocker azilsartan medoxomil vs valsartan by ambulatory blood pressure monitoring. J Clin Hypertens (Greenwich). 2011;13(7):467-472.

13. Bowlus WE, Langford HG. A comparison of the antihypertensive effect of chlorthalidone and hydrochlorothiazide. Clin Pharmacol Ther. 1964;5:708-711.

14. Riess W, Dubach UC, Burckhardt D, Theobald W, Vuillard P, Zimmerli M. Pharmacokinetic studies with chlorthalidone (Hygroton) in man. Eur J Clin Pharmacol. 1977;12(5):375-382.

15. McAinsh J, Bastain W, Young J, Harry JD. Bioavailability in man of atenolol and chlorthalidone from a combination formulation. Biopharm Drug Dispos. 1981;2(2):147-156. 
16. Collste $P$, Garle M, Rawlins MD, Sjöqvist F. Interindividual differences in chlorthalidone concentration in plasma and red cells of man after single and multiple doses. Eur J Clin Pharmacol. 1976;9(4):319-325.

17. Bengtsson C, Johnsson G, Sannerstedt R, Werko L. Effect of different doses of chlorthalidone on blood pressure, serum potassium, and serum urate. Br Med J. 1975;1(5951):197-199.

18. Takeda Pharmaceuticals USA, Inc. Prescribing Information: Edarbyclor. Deerfield: Takeda Pharmaceuticals America, Inc; 2012. Available from: http://www.edarbi.com/. Accessed January 3, 2013.

19. Mortality after $101 / 2$ years for hypertensive participants in the Multiple Risk Factor Intervention Trial. Circulation. 1990;82(5):1616-1628.

20. Dorsch MP, Gillespie BW, Erickson SR, Bleske BE, Weder AB. Chlorthalidone reduces cardiovascular events compared with hydrochlorothiazide: a retrospective cohort analysis. Hypertension. 2011;57(4):689-694.

21. Sica D, Bakris GL, White WB, et al. Blood pressure lowering efficacy of the fixed dose combination of azilsartan and chlorthalidone: a factorial study. J Clin Hypertens (Greenwich). 2012;14(5):284-292.

22. Cushman WC, Bakris GL, White WB, et al. Azilsartan medoxomil plus chlorthalidone reduces blood pressure more effectively than olmesartan plus hydrochlorthiazide in stage 2 systolic hypertension. Hypertension. 2012;60(2):310-318.

23. Bakris GL, Sica D, White WB, et al. Antihypertensive efficacy of hydrochlorothiazide vs chlorthalidone combined with azilsartan medoxomil. Am J Med. 2012;125(12):1229. e1-1229. e10.

24. Divisional Memo: Azilsartan medoxomil (Edarbi) for hypertension. Center for Drug Evaluation and Research. Medical Review(s). Silver Spring, MD, USA: Food and Drug Administration, 2011. Available from: http:/www.accessdata.fda.gov/drugsatfda_docs/ nda/2011/200796Orig1s000MedR.pdf. Accessed April 12, 2013.

25. Addendum to Clinical Review of NDA 202-331 (Edarbyclor ${ }^{\sqrt{ }}$ ). Center for Drug Evaluation and Research. Medical Review(s). Silver Spring, MD, USA: Food and Drug Administration, 2011. Available from: http://www. accessdata.fda.gov/drugsatfda_docs/nda/2011/202331Orig1s000MedR. pdf. Accessed January 3, 2013.
26. Medication Average Wholesale Price. Thomson Reuters Micromedex Clinical Evidence Solutions [Internet]. Thomson Reuters; c2011. RED BOOK Drug References; c2011 [cited July 8, 2011]. Available from: http://thomsonreuters.com/products_services/healthcare/healthcare_ products/clinical_deci_support/micromedex_clinical_evidence_sols/ med_safety_solutions/red_book/. Accessed April 13, 2013.

27. Cushman WC, Ford CE, Cutler JA, et al; ALLHAT Collaborative Research Group. Success and predictors of blood pressure control in diverse North American settings: the antihypertensive and lipid-lowering treatment to prevent heart attack trial (ALLHAT). J Clin Hypertens (Greenwich). 2002;4(6):393-404.

28. Dahlöf B, Devereux RB, Kjeldsen SE, et al; LIFE Study Group. Cardiovascular morbidity and mortality in the Losartan Intervention For Endpoint reduction in hypertension study (LIFE): a randomized trial against atenolol. Lancet. 2002;359(9311):995-1003.

29. Pepine CJ, Handberg EM, Cooper-DeHoff RM, et al; INVEST Investigators. A calcium antagonist vs a non-calcium antagonist hypertension treatment strategy for patients with coronary artery disease. The International Verapamil-Trandolapril Study (INVEST): a randomized controlled trial. JAMA. 2003;290(21):2805-2816.

30. Dahlöf B, Sever PS, Poulter N, et al; ASCOT Investigators. Prevention of cardiovascular events with an antihypertensive regimen of amlodipine adding perindopril as required versus atenolol adding bendroflumethiaizde as required in the Ango-Scandinavian Cardiac Outcomes Trial-Blood Pressure Lowering Arm (ASCOT-BPLA): a multicentre randomized controlled trial. Lancet. 2005; 366(9489): 895-906.

31. Weber MA, Julius S, Kjeldsen SE, et al. Blood pressure dependent and independent effects of antihypertensive treatment on clinical events in the VALUE Trial. Lancet. 2004;363(9426):2049-2051.

32. Corrao G, Nicotra F, Parodi A, et al. Cardiovascular protection by initial and subsequent combination of antihypertensive drugs in daily life practice. Hypertension. 2011;58(4):566-572.
Integrated Blood Pressure Control

\section{Publish your work in this journal}

Integrated Blood Pressure Control is an international, peer-reviewed open-access journal focusing on the integrated approach to managing hypertension and risk reduction. Treating the patient and comorbidities together with diet and lifestyle modification and optimizing healthcare resources through a multidisciplinary team approach constitute key

\section{Dovepress}

features of the journal. This journal is indexed on American Chemical Society's Chemical Abstracts Service (CAS). The manuscript management system is completely online and includes a very quick and fair peerreview system, which is all easy to use. Visit http://www.dovepress.com/ testimonials.php to read real quotes from published authors 